\title{
DÜBLIN
}

Technological University Dublin

ARROW@TU Dublin

2010-01-01

\section{Economic Risk Assessment using the Fractal Market Hypothesis}

Jonathan Blackledge

Technological University Dublin, jonathan.blackledge@tudublin.ie

Marek Rebow

Technological University Dublin, marek.rebow@tudublin.ie

Follow this and additional works at: https://arrow.tudublin.ie/engscheleart

Part of the Applied Statistics Commons, Finance and Financial Management Commons, Management Sciences and Quantitative Methods Commons, Probability Commons, and the Statistical Models

Commons

\section{Recommended Citation}

Blackledge, J., Rebow, M.:Economic Risk Assessment using the Fractal Market Hypothesis. Fifth International Conference on Internet Monitoring and Protection, Barcelona, pp.41-47.

This Conference Paper is brought to you for free and open access by the School of Electrical and Electronic Engineering at ARROW@TU Dublin. It has been accepted for inclusion in Conference papers by an authorized administrator of ARROW@TU Dublin. For more information, please contact arrow.admin@tudublin.ie, aisling.coyne@tudublin.ie,gerard.connolly@tudublin.ie.

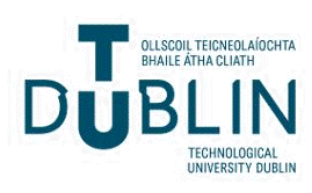




\section{Economic Risk Assessment using the Fractal Market Hypothesis}

\author{
J. M. Blackledge \\ School of Electrical Engineering Systems, \\ College of Engineering \\ and the Built Environment, \\ Dublin Institute of Technology, \\ Dublin, Ireland. \\ Email: jonathan.blackledge@dit.ie
}

\author{
M. Rebow \\ College of Engineering \\ and the Built Environment, \\ Dublin Institute of Technology, \\ Dublin, Ireland, \\ Email:marek.rebow@dit.ie
}

\begin{abstract}
This paper considers the Fractal Market Hypothesis (FMH) for assessing the risk(s) in developing a financial portfolio based on data that is available through the Internet from an increasing number of sources. Most financial risk management systems are still based on the Efficient Market Hypothesis which often fails due to the inaccuracies of the statistical models that underpin the hypothesis, in particular, that financial data are based on stationary Gaussian processes. The FMH considered in this paper assumes that financial data are non-stationary and statistically self-affine so that a risk analysis can, in principal, be applied at any time scale provided there is sufficient data to make the output of a FMH analysis statistically significant.
\end{abstract}

Keywords-Risk assessment of economy, Risk assessment statistics and numerical data, Fractal Market Hypothesis, ABX index.

\section{INTRODUCTION}

Attempts to develop stochastic models for financial time series, which are essentially digital signals composed of 'tick data' $^{1}$ [1], [2] can be traced back to the early Twentieth Century when Louis Bachelier [3] proposed that fluctuations in the prices of stocks and shares (which appeared to be yesterday's price plus some random change) could be viewed in terms of random walks in which price changes were entirely independent of each other. Thus, one of the simplest models for price variation is based on the sum of independent random numbers. This is the basis for Brownian motion [4] in which the random numbers are considered to conform to a normal distribution.

\section{Brownian Motion AND the EFficient Market HYPOTHESIS}

Random walk models, which underpin the so called Efficient Market Hypothesis (EMH) [5]-[12], have been the basis for financial time series analysis since the work of Bachelier in the late Nineteenth Century. Although the Black-Scholes equation [13], developed in the 1970s for valuing options, is deterministic (one of the first financial

\footnotetext{
${ }^{1}$ Data that provides traders with daily tick-by-tick data - time and sales - of trade price, trade time, and volume traded, for example, at different sampling rates as required.
}

models to achieve determinism), it is still based on the EMH, i.e. stationary Gaussian statistics. The EMH is based on the principle that the current price of an asset fully reflects all available information relevant to it and that new information is immediately incorporated into the price. Thus, in an efficient market, the modelling of asset prices is concerned with modelling the arrival of new information. New information must be independent and random, otherwise it would have been anticipated and would not be new. The arrival of new information can send 'shocks' through the market (depending on the significance of the information) as people react to it and then to each other's reactions. The EMH assumes that there is a rational and unique way to use the available information and that all agents possess this knowledge. Further, the EMH assumes that this 'chain reaction' happens effectively instantaneously. These assumptions are clearly questionable at any and all levels of a complex financial system.

The EMH implies independence of price increments and is typically characterised by a normal of Gaussian Probability Density Function (PDF) which is chosen because most price movements are presumed to be an aggregation of smaller ones, the sums of independent random contributions having a Gaussian PDF. However, it has long been known that financial time series do not follow random walks. The shortcomings of the EMH model include: failure of the independence and Gaussian distribution of increments assumption, clustering, apparent non-stationarity and failure to explain momentous financial events such as 'crashes' leading to recession and, in some extreme cases, depression. These limitations have prompted a new class of methods for investigating time series obtained from a range of disciplines. For example, Re-scaled Range Analysis (RSRA), e.g. [14]-[16], which is essentially based on computing the Hurst exponent [17], is a useful tool for revealing some well disguised properties of stochastic time series such as persistence (and anti-persistence) characterized by non-periodic cycles. Non-periodic cycles correspond to trends that persist for irregular periods but with a degree of statistical regularity often associated with non-linear dynamical systems. RSRA 
is particularly valuable because of its robustness in the presence of noise. The principal assumption associated with RSRA is concerned with the self-affine or fractal nature of the statistical character of a time-series rather than the statistical 'signature' itself. Ralph Elliott first reported on the fractal properties of financial data in 1938 (e.g. [18] and reference therein). He was the first to observe that segments of financial time series data of different sizes could be scaled in such a way that they were statistically the same producing so called Elliot waves.

\section{RISK ASSESSMENT AND REPEATING ECONOMIC PATTERNS}

A good stochastic financial model should ideally consider all the observable behaviour of the financial system it is attempting to model. It should therefore be able to provide some predictions on the immediate future behaviour of the system within an appropriate confidence level. Predicting the markets has become (for obvious reasons) one of the most important problems in financial engineering. Although, at least in principle, it might be possible to model the behaviour of each individual agent operating in a financial market, one can never be sure of obtaining all the necessary information required on the agents themselves and their modus operandi. This principle plays an increasingly important role as the scale of the financial system, for which a model is required, increases. Thus, while quasi-deterministic models can be of value in the understanding of micro-economic systems (with known 'operational conditions'), in an ever increasing global economy (in which the operational conditions associated with the fiscal policies of a given nation state are increasingly open), we can take advantage of the scale of the system to describe its behaviour in terms of functions of random variables.

\section{A. Elliot Waves}

The stochastic nature of financial time series is well known from the values of the stock market major indices such as the FTSE (Financial Times Stock Exchange) in the UK, the Dow Jones in the US which are frequently quoted. A principal aim of investors is to attempt to obtain information that can provide some confidence in the immediate future of the stock markets often based on patterns of the past. One of the principal components of this aim is based on the observation that there are 'waves within waves' and 'events within events' that appear to permeate financial signals when studied with sufficient detail and imagination. It is these repeating patterns that occupy both the financial investor and the systems modeller alike and it is clear that although economies have undergone many changes in the last one hundred years, the dynamics of market data do not appear to change significantly (ignoring scale). For example, Figure 1 shows the re-scaled 'macrotrends' (i.e. normalised Gaussian

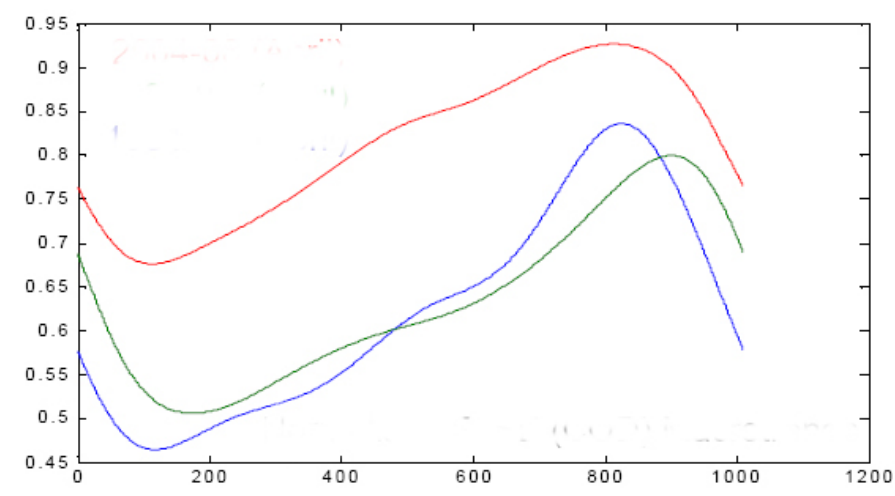

Figure 1. Evolution of the 1987, 1997 and 2007 financial crashes. Normalised macrotrends (i.e. where the data has been smoothed and rescaled to values between 0 and 1 inclusively) of the daily FTSE value (close-of-day) for 02-04-1984 to 24-12-1987 (blue), 05-04-1994 to 24-121997 (green) and 02-04-2004 to 24-09-2007 (red).

lowpass filtered signals) associated with FTSE Close-ofDay (COD) illustrating the 'development' of three different 'crashes', the one of 1987 and that of 1997 and the most recent crash of 2007. The similarity in behaviour of these signals is remarkable and clearly indicates a wavelength of approximately 1000 days. This is indicative of the quest to understand economic signals in terms of some universal phenomenon from which appropriate (macro) economic models can be generated. In an efficient market, only the revelation of some dramatic information can cause a crash, yet postmortem analysis of crashes typically fail to (convincingly) tell us what this information must have been.

One cause of correlations in market price changes (and volatility) is mimetic behaviour, known as herding. In general, market crashes happen when large numbers of agents place sell orders simultaneously creating an imbalance to the extent that market makers are unable to absorb the other side without lowering prices substantially. Most of these agents do not communicate with each other, nor do they take orders from a leader. In fact, most of the time they are in disagreement, and submit roughly the same amount of buy and sell orders. This is a healthy non-crash situation; it is a diffusive (random-walk) process which underlies the $\mathrm{EMH}$ and financial portfolio rationalization.

\section{B. Non-equilibrium Systems}

Financial markets can be considered to be non-equilibrium systems because they are constantly driven by transactions that occur as the result of new fundamental information about firms and businesses. They are complex systems because the market also responds to itself, often in a highly non-linear fashion, and would carry on doing so (at least for some time) in the absence of new information. The 'price change field' is highly non-linear and very sensitive to exogenous shocks and it is probable that all shocks have a long term effect. Market transactions generally occur 
globally at the rate of hundreds of thousands per second. It is the frequency and nature of these transactions that dictate stock market indices, just as it is the frequency and nature of the sand particles that dictates the statistics of the avalanches in a sand pile. These are all examples of random scaling fractals [19]-[24].

\section{The Fractal MARKET Hypothesis}

Developing mathematical models to simulate stochastic processes has an important role in financial analysis and information systems in general where it should be noted that information systems are now one of the most important aspects in terms of regulating financial systems, e.g. [25][28]. A good stochastic model is one that accurately predicts the statistics we observe in reality, and one that is based upon some well defined rationale. Thus, the model should not only describe the data, but also help to explain and understand the system.

There are two principal criteria used to define the characteristics of a stochastic field: (i) The PDF or the Characteristic Function (i.e. the Fourier transform of the PDF); the Power Spectral Density Function (PSDF). The PSDF is the function that describes the envelope or shape of the power spectrum of a signal. In this sense, the PSDF is a measure of the field correlations. The PDF and the PSDF are two of the most fundamental properties of any stochastic field and various terms are used to convey these properties. For example, the term 'zero-mean white Gaussian noise' refers to a stochastic field characterized by a PSDF that is effectively constant over all frequencies (hence the term 'white' as in 'white light') and has a PDF with a Gaussian profile whose mean is zero.

Stochastic fields can of course be characterized using transforms other than the Fourier transform (from which the PSDF is obtained) but the conventional PDF-PSDF approach serves many purposes in stochastic systems theory. However, in general, there is no general connectivity between the PSDF and the PDF either in terms of theoretical prediction and/or experimental determination. It is not generally possible to compute the PSDF of a stochastic field from knowledge of the PDF or the PDF from the PSDF. Hence, in general, the PDF and PSDF are fundamental but nonrelated properties of a stochastic field. However, for some specific statistical processes, relationships between the PDF and PSDF can be found, for example, between Gaussian and non-Gaussian fractal processes [29] and for differentiable Gaussian processes [30].

There are two conventional approaches to simulating a stochastic field. The first of these is based on predicting the PDF (or the Characteristic Function) theoretically (if possible). A pseudo random number generator is then designed whose output provides a discrete stochastic field that is characteristic of the predicted PDF. The second approach is based on considering the PSDF of a field which, like the
PDF, is ideally derived theoretically. The stochastic field is then typically simulated by filtering white noise. A 'good' stochastic model is one that accurately predicts both the PDF and the PSDF of the data. It should take into account the fact that, in general, stochastic processes are non-stationary. In addition, it should, if appropriate, model rare but extreme events in which significant deviations from the norm occur.

One explanation for crashes involves a replacement for the EMH by the Fractal Market Hypothesis (FMH) which is the basis of the model considered in this paper. The FMH proposes the following: (i) The market is stable when it consists of investors covering a large number of investment horizons which ensures that there is ample liquidity for traders; (ii) information is more related to market sentiment and technical factors in the short term than in the long term - as investment horizons increase and longer term fundamental information dominates; (iii) if an event occurs that puts the validity of fundamental information in question, long-term investors either withdraw completely or invest on shorter terms (i.e. when the overall investment horizon of the market shrinks to a uniform level, the market becomes unstable); (iv) prices reflect a combination of short-term technical and long-term fundamental valuation and thus, short-term price movements are likely to be more volatile than long-term trades - they are more likely to be the result of crowd behaviour; ( $v$ ) if a security has no tie to the economic cycle, then there will be no long-term trend and short-term technical information will dominate. Unlike the $\mathrm{EMH}$, the FMH states that information is valued according to the investment horizon of the investor. Because the different investment horizons value information differently, the diffusion of information will also be uneven. Unlike most complex physical systems, the agents of the economy, and perhaps to some extent the economy itself, have an extra ingredient, an extra degree of complexity. This ingredient is consciousness.

\section{Mathematical Model for the FMH}

We consider an economic times series to be a solution to the fractional diffusion equation [31]-[36]

$$
\left(\frac{\partial^{2}}{\partial x^{2}}-\sigma^{q} \frac{\partial^{q}}{\partial t^{q}}\right) u(x, t)=\delta(x) n(t)
$$

where $\sigma$ is the fractional diffusion coefficient and $q>0$ is the 'Fourier dimension' and $n(t)$ is 'white noise' Let

$$
u(x, t)=\frac{1}{2 \pi} \int_{-\infty}^{\infty} U(x, \omega) \exp (i \omega t) d \omega
$$

and

$$
n(t)=\frac{1}{2 \pi} \int_{-\infty}^{\infty} N(\omega) \exp (i \omega t) d \omega .
$$


Then, using the result

$$
\frac{\partial^{q}}{\partial t^{q}} u(x, t)=\frac{1}{2 \pi} \int_{-\infty}^{\infty} U(x, \omega)(i \omega)^{q} \exp (i \omega t) d \omega
$$

we can transform the fractional diffusion equation to the form

$$
\left(\frac{\partial^{2}}{\partial x^{2}}+\Omega_{q}^{2}\right) U(x, \omega)=f(x) N(\omega)
$$

where we take

$$
\Omega_{q}=i(i \omega \sigma)^{\frac{q}{2}}
$$

and ignore the case for $\Omega_{q}=-i(i \omega \sigma)^{\frac{q}{2}}$. Defining the Green's function $g$ [37] to be the solution of

$$
\left(\frac{\partial^{2}}{\partial x^{2}}+\Omega_{q}^{2}\right) g\left(x \mid x_{0}, \omega\right)=\delta\left(x-x_{0}\right)
$$

where $\delta$ is the delta function, we obtain the following solution:

$$
\begin{aligned}
U\left(x_{0}, \omega\right) & =N(\omega) \int_{-\infty}^{\infty} g\left(x \mid x_{0}, \omega\right) \delta(x) d x \\
& =N(\omega) g\left(\left|x_{0}\right|, \omega\right)
\end{aligned}
$$

where [38]

$$
g\left(x \mid x_{0}, k\right)=\frac{i}{2 \Omega_{q}} \exp \left(i \Omega_{q}\left|x-x_{0}\right|\right)
$$

under the assumption that $u$ and $\partial u / \partial x \rightarrow 0$ as $x \rightarrow \pm \infty$. Simplification of this result can be obtained by considering the frequency response of $U$ close to the origin $x_{0}$. Thus as $x_{0} \rightarrow 0$

$$
U(\omega)=\frac{i N(\omega)}{2 \Omega_{q}}=\frac{1}{2 \sigma^{\frac{q}{2}}} \frac{N(\omega)}{(i \omega)^{\frac{q}{2}}}
$$

The time series if then given by Fourier inverting this equation to give (ignoring scaling parameters)

$$
u(t)=\frac{1}{t^{1-q / 2}} \otimes n(t)
$$

This equation is the RiemannLiouville transform (ignoring scaling) which is a fractional integral and defines a random scaling fractal signal $u(t)$.

\section{A. Rationale for the Model}

Hurst process describe fractional Brownian motion and are based on the generalization of Brownian motion quantified by the equation $A(t)=a \sqrt{t}$ to

$$
A(t)=a t^{H}, \quad H \in(0,1]
$$

where $a$ is the length of each random step in the plane and $A$ is the most likely position in the plane after time $t$ with respect to the position at $t=0$. Given that random walks with $H=0.5$ describe processes whose macroscopic behaviour is characterised by the diffusion equation, then, by induction, Hurst processes should be characterised by generalizing the diffusion operator

$$
\frac{\partial^{2}}{\partial x^{2}}-\sigma \frac{\partial}{\partial t}
$$

to the fractional form

$$
\frac{\partial^{2}}{\partial x^{2}}-\sigma^{q} \frac{\partial^{q}}{\partial t^{q}}
$$

where $q \in(0,2]$ and $D=1 / \sigma$ is the fractional diffusivity. Fractional diffusive processes can therefore be interpreted as intermediate between classical diffusive (random phase walks with $H=0.5$; diffusive processes with $q=1$ ) and 'propagative process' (coherent phase walks for $H=1$; propagative processes with $q=2$ ), e.g. [39]-[41].

\section{B. Non-stationary Model}

The fractional diffusion operator given above is appropriate for modelling fractional diffusive processes that are stationary. For non-stationary fractional diffusion, we could consider the case where the diffusivity is time variant as defined by the function $\sigma(t)$. However, a more interesting case arises when the characteristics of the diffusion processes change over time becoming less or more diffusive. This is illustrated in terms of the random walk in the plane given in Figure 2. Here, the walk starts off being fully diffusive (i.e. $H=0.5$ and $q=1$ ), changes to being fractionally diffusive $(0.5<H<1$ and $1<q<2)$ and then changes back to being fully diffusive. In terms of fractional diffusion, this is equivalent to having an operator

$$
\frac{\partial^{2}}{\partial x^{2}}-\sigma^{q} \frac{\partial^{q}}{\partial t^{q}}
$$

where $q=1, t \in\left(0, T_{1}\right] ; q>1, t \in\left(T_{1}, T_{2}\right] ; q=1, t \in$ $\left(T_{2}, T_{3}\right]$ where $T_{3}>T_{2}>T_{1}$. If we want to generalise such processes over arbitrary periods of time, then we should consider $q$ to be a function of time. We can then introduce a non-stationary fractional diffusion operator given by

$$
\frac{\partial^{2}}{\partial x^{2}}-\sigma^{q(t)} \frac{\partial^{q(t)}}{\partial t^{q(t)}}
$$

This operator is the theoretical basis for the Fractal Market Hypothesis considered in this paper.

If we consider the case where the Fourier dimension is a relatively slowly varying function of time, then we can legitimately consider $q(t)$ to be composed of a sequence of different states $q_{i}=q\left(t_{i}\right)$. This approach allows us to develop a stationary solution for a fixed $q$ over a fixed period of time. Non-stationary behaviour can then be introduced by using the same solution for different values of $q$ over fixed (or varying) periods of time and concatenating the solutions for all $q$. 


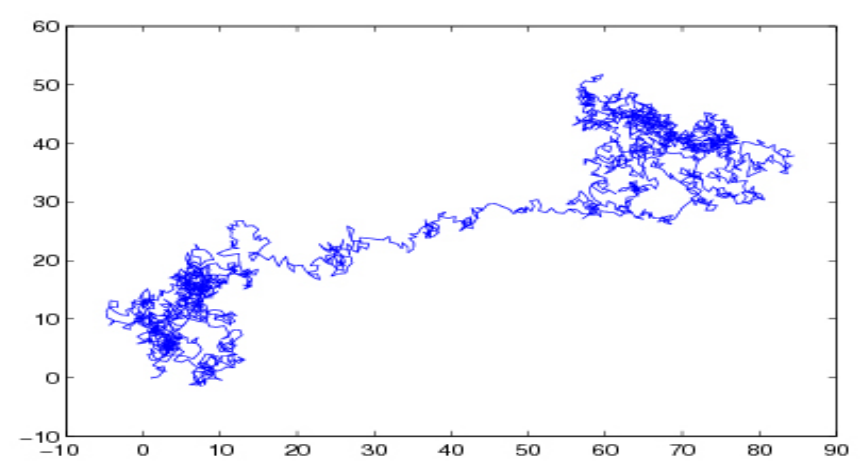

Figure 2. Non-stationary random phase walk in the plane.

\section{Financial Data Analysis}

We consider the basic model for a quasi-stationary segment of a financial signal to be given by

$$
u(t)=\frac{1}{t^{1-q / 2}} \otimes n(t), \quad q>0
$$

which has characteristic spectrum

$$
U(\omega)=\frac{N(\omega)}{(i \omega)^{q / 2}}
$$

The PSDF is thus characterised by $\omega^{-q}, \omega \geq 0$ and our problem is thus, to compute $q$ from the data $P(\omega)=1$ $\left.U(\omega)\right|^{2}, \omega \geq 0$. For this data, we consider the PSDF

$$
\hat{P}(\omega)=\frac{c}{\omega^{q}}
$$

or

$$
\ln \hat{P}(\omega)=C+q \ln \omega
$$

where $C=\ln c$. The problem is therefore reduced to implementing an appropriate method to compute $q$ (and C) by finding a best fit of the line $\ln \hat{P}(\omega)$ to the data $\ln P(\omega)$. Application of the least squares method for computing $q$, which is based on minimizing the error

$$
e(q, C)=\|\ln P(\omega)-\ln \hat{P}(\omega, q, C)\|_{2}^{2}
$$

with regard to $q$ and $C$, leads to errors in the estimates for $q$ which are not compatible with market data analysis. The reason for this is that relative errors at the start and end of the data $\ln P$ may vary significantly especially because any errors inherent in the data $P$ will be 'amplified' through application of the logarithmic transform required to linearise the problem. In general, application of a least squares approach is very sensitive to statistical heterogeneity [42] and in this application, may provide values of $q$ that are not compatible with the rationale associated with the FMH (i.e. values of $1<q<2$ that are intermediate between diffusive and propagative processes). For this reason, an alternative approach must be considered which, in this paper, is based on Orthogonal Linear Regression (OLR).

\section{A. Moving Window Data Analysis}

Applying a standard moving window, $q(t)$ is computed by repeated application of OLR based on the m-code available from [43]. This provides a numerical estimate of the function $q(t)$ whose values reflect the state of a financial signals (assumed to be a non-stationary random fractal) in terms of a stable or unstable economy, from which a risk analysis can be performed. Since $q$ is, in effect, a statistic, its computation is only as good as the quantity (and quality) of data that is available for its computation. For this reason, a relatively large window is required whose length is compatible with the number of samples available.

The approach has been software engineered using MATLAB to develop a prototype system that is currently available at http://eleceng.dit.ie/arg/downloads/FMH.zip The system has been designed to use financial data that is readily available from Yahoo finance http://uk.finance.yahoo.com/.

An example of the system output using a 1024 element window is given in Figure 3 which includes $q(t)$ after it has been smoothed using a Gaussian low-pass filter to reveal the underlying macrotrends of $q(t)$. Inspection of the signals illustrates a qualitative relationship between trends in the financial data and $q(t)$ in accordance with the theoretical model considered. In particular, over periods of time in which $q$ increases in value, the amplitude of the financial signal $u(t)$ decreases. Moreover, and more importantly, an upward trend in $q$ appears to be a pre-cursur to a downward trend in $u(t)$, a correlation that is compatible with the idea that a rise in the value of $q$ relates to the 'system' becoming more propagative, which in stock market terms, indicates the likelihood for the markets becoming 'bear' dominant in the future.

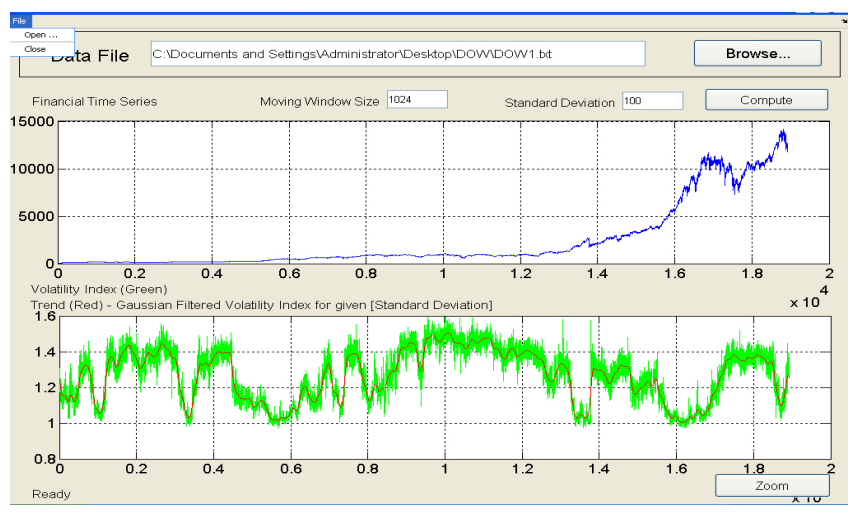

Figure 3. Application of the FMH using a 1024 element window for analysing financial time series composed of Dow Jones close-of-day values from 01-10-1928 to 25-03-2009 The plot shows the Dow Jones data (blue signal above) and the time varying Fourier Dimension $q(t)$ (green signal below) onto which is superimposed a Gaussian low-pass filtered version of the signal (red). 
The results of using the method discussed above not only provides for a general appraisal of different macroeconomic financial time series, but, with regard to the size of selected window used, an analysis of data at any point in time. The output can be interpreted in terms of 'persistence' and 'anti-persistence' and in terms of the existence or absence of after-effects (macroeconomic memory effects). For those periods in time when $q(t)$ is relatively constant, the existing market tendencies usually remain. Changes in the existing trends tend to occur just after relatively sharp changes in $q(t)$ have developed. This behaviour indicates the possibility of using the time series $q(t)$ for identifying the behaviour of a macroeconomic financial system in terms of both inter-market and between-market analysis. These results support the possibility of using $q(t)$ as an independent macroeconomic volatility predictor.

\section{DISCUSSION}

The non-stationary nature of the model presented in this paper is taken to account for stochastic processes that can vary in time and are intermediate between diffusive and propagative or persistent behaviour. Application of Orthogonal Linear Regression to macroeconomic time series data provides an accurate and robust method to compute $q(t)$ when compared to other statistical estimation techniques such as the least squares method. As a result of the physical interpretation associated with the fractional diffusion equation and the 'meaning' of $q(t)$, we can, in principal, use the signal $q(t)$ as a predictive measure in the sense that as the value of $q(t)$ continues to increases, there is a greater likelihood for volatile behaviour of the markets. This is reflected in the data analysis based on the example given in Figure 3 and other financial data, the results of which lie beyond the scope of this paper ${ }^{2}$. It should be noted that because financial time series data is assumed to be selfaffine, the computation of $q(t)$ can be applied over any time scale.

In a statistical sense, $q(t)$ is just another measure that may, or otherwise, be of value to market traders. In comparison with other statistical measures, this can only be assessed through its practical application in a live trading environment. However, in terms of its relationship to a stochastic model for macroeconomic data, $q(t)$ does provide a measure that is consistent with the physical principles associated with a random walk that includes a directional bias, i.e. fractional Brownian motion. The model considered, and the signal processing algorithm proposed, has a close association with re-scaled range analysis for computing the Hurst exponent $H$ [33]. In this sense, the principal contribution of this paper has been to consider a model that is quantified in terms of a physically significant (but phenomenological) model that is compounded in a specific (fractional) partial differential

\footnotetext{
${ }^{2}$ Similar results being observed for other major stock markets.
}

equation. As with other financial time series, their derivatives, transforms etc., a range of statistical measures can be used to characterise $q(t)$, an example being given in Figure 3 where $q(t)$ has been smoothed to provide a measure of the underlying trends.

\section{CONCLUSION}

In terms of the non-stationary fractional diffusive model considered in this work, the time varying Fourier dimension $q(t)$ can be interpreted in terms of a 'gauge' on the characteristics of a dynamical system. This includes the management processes from which all modern economies may be assumed to be derived. In this sense, the FMH is based on three principal considerations: (i) the non-stationary behaviour associated with any system undergoing continuous change that is driven by a management infrastructure; (ii) the cause and effect that is inherent at all scales (i.e. all levels of management hierarchy); (iii) the self-affine nature of outcomes relating to points (i) and (ii). In a modern economy, the principal issue associated with any form of financial management is based on the flow of information and the assessment of this information at different points connecting a large network. In this sense, a macroeconomy can be assessed in terms of its information network which consists of a distribution of nodes from which information can flow in and out. The 'efficiency' of the system is determined by the level of randomness associated with the direction of flow of information to and from each node. The nodes of the system are taken to be individuals or small groups of individuals whose assessment of the information they acquire together with their remit, responsibilities and initiative, determines the direction of the information flow from one node to the next. The determination of the efficiency of a system in terms of randomness is the most critical in terms of the model developed. It suggests that the performance of a business is related to how well information flows through an organisation.

\section{ACKNOWLEDGMENT}

The author is grateful for the support of the Science Foundation Ireland and Dublin Institute of Technology.

\section{REFERENCES}

[1] http://www.tickdata.com/

[2] http://www.vhayu.com/

[3] http://en.wikipedia.org/wiki/Louis_Bachelier

[4] http://en.wikipedia.org/wiki/Robert_Brown_(botanist)

[5] T. R. Copeland, J. F. Weston and K. Shastri, Financial Theory and Corporate Policy, 4th Edition, Pearson Addison Wesley, 2003. 
[6] J. D. Martin, S. H. Cox, R. F. McMinn and R. D. Maminn, The Theory of Finance: Evidence and Applications, International Thomson Publishing, 1997.

[7] R. C. Menton, Continuous-Time Finance, Blackwell Publishers, 1992.

[8] T. J. Watsham and K. Parramore, Quantitative Methods in Finance, Thomson Business Press, 1996.

[9] E. Fama, The Behavior of Stock Market Prices, Journal of Business Vol. 38, 34-105, 1965.

[10] P. Samuelson, Proof That Properly Anticipated Prices Fluctuate Randomly, Industrial Management Review Vol. 6, 41-49, 1965.

[11] E. Fama, Efficient Capital Markets: A Review of Theory and Empirical Work, Journal of Finance Vol. 25, 383-417, 1970.

[12] G. M. Burton, Efficient Market Hypothesis, The New Palgrave: A Dictionary of Economics, Vol. 2, 120-23, 1987.

[13] F. Black and M. Scholes, The Pricing of Options and Corporate Liabilities, Journal of Political Economy, Vol. 81(3), 637-659, 1973.

[14] http://uk.finance.yahoo.com/q/hp?s=\%5EFTSE

[15] B. B. Mandelbrot and J. R. Wallis, Robustness of the Rescaled Range $R / S$ in the Measurement of Noncyclic Long Run Statistical Dependence, Water Resources Research, Vol. 5(5), 967-988, 1969.

[16] B. B. Mandelbrot, Statistical Methodology for Non-periodic Cycles: From the Covariance to R/S Analysis, Annals of Economic and Social Measurement, Vol. 1(3), 259-290, 1972.

[17] E. H. Hurst, A Short Account of the Nile Basin, Cairo, Government Press, 1944.

[18] http://en.wikipedia.org/wiki/Elliott_wave_principle

[19] B. B. Mandelbrot, The Fractal Geometry of Nature, Freeman, 1983.

[20] J. Feder, Fractals, Plenum Press, 1988.

[21] K. J. Falconer, Fractal Geometry, Wiley, 1990.

[22] P. Bak, How Nature Works, Oxford University Press, 1997.

[23] N. Lam and L. De Cola L, Fractal in Geography, PrenticeHall, 1993.

[24] H. O. Peitgen and D. Saupe (Eds.), The Science of Fractal Images, Springer, 1988.

[25] A. J. Lichtenberg and M. A. Lieberman, Regular and Stochastic Motion: Applied Mathematical Sciences, Springer-Verlag, 1983.

[26] J. J. Murphy, Intermarket Technical Analysis: Trading Strategies for the Global Stock, Bond, Commodity and Currency Market, Wiley Finance Editions, Wiley, 1991.
[27] J. J. Murphy, Technical Analysis of the Futures Markets: A Comprehensive Guide to Trad-ing Methods and Applications, New York Institute of Finance, Prentice-Hall, 1999.

[28] T. R. DeMark, The New Science of Technical Analysis, Wiley, 1994.

[29] J. O. Matthews, K. I. Hopcraft, E. Jakeman and G. B. Siviour, Accuracy Analysis of Measurements on a Stable Power-law Distributed Series of Events, J. Phys. A: Math. Gen. 39, 1396713982, 2006.

[30] W. H. Lee, K. I. Hopcraft, and E. Jakeman, Continuous and Discrete Stable Processes, Phys. Rev. E 77, American Physical Society, 011109, 1-4.

[31] A. Einstein, On the Motion of Small Particles Suspended in Liquids at Rest Required by the Molecular-Kinetic Theory of Heat, Annalen der Physik, Vol. 17, 549-560, 1905.

[32] J. M. Blackledge, G. A. Evans and P. Yardley, Analytical Solutions to Partial Differential Equations, Springer, 1999.

[33] H. Hurst, Long-term Storage Capacity of Reservoirs, Transactions of American Society of Civil Engineers, Vol. 116, 770-808, 1951.

[34] M. F. Shlesinger, G. M. Zaslavsky and U. Frisch (Eds.), Lévy Flights and Related Topics in Physics, Springer 1994.

[35] R. Hilfer, Foundations of Fractional Dynamics, Fractals Vol. 3(3), 549-556, 1995.

[36] A. Compte, Stochastic Foundations of Fractional Dynamics, Phys. Rev E, Vol. 53(4), 4191-4193, 1996.

[37] P. M. Morse and H. Feshbach, Methods of Theoretical Physics, McGraw-Hill, 1953.

[38] G. F. Roach, Green's Functions (Introductory Theory with Applications), Van Nostrand Reihold, 1970.

[39] T. F. Nonnenmacher, Fractional Integral and Differential Equations for a Class of Lévy-type Probability Densities, J. Phys. A: Math. Gen. Vol. 23, L697S-L700S, 1990.

[40] R. Hilfer, Exact Solutions for a Class of Fractal Time Random Walks, Fractals, Vol. 3(1), 211-216, 1995.

[41] R. Hilfer and L. Anton, Fractional Master Equations and Fractal Time Random Walks, Phys. Rev. E, Vol. 51(2), R848R851, 1995.

[42] I. Lvova, Application of Statistical Fractional Methods for the Analysis of Time Series of Currency Exchange Rates, $\mathrm{PhD}$ Thesis, De Montfort University, 2006.

[43] http://www.mathworks.com/matlabcentral/fileexchange/ loadFile.do?objectId=6716\&objectType $=$ File 